This is an Accepted Manuscript of an article published by Science Reviews 2000 Ltd in Science Progress on 01 September 2016, available online: http:// dx.doi.org/10.3184/003685016x14670237050493 


\title{
Side-Chain Liquid Crystal Conducting Polymers
}

\author{
DANESH ROUDINI and PETER J.S. FOOT \\ Materials Research Centre and School of Life Sciences, Pharmacy and Chemistry \\ SEC Faculty, Kingston University London, Kingston upon Thames KT1 2EE, U.K. \\ * P.J.Foot@kingston.ac.uk \\ * Roudini.Danesh@yahoo.com
}

\begin{abstract}
This article overviews some of the research progress on the effects of incorporating liquid crystalline functionalities into commercial conjugated polymers such as polythiophene, polypyrrole and polyaniline. Polarisable aromatic mesogens are generally attached via a flexible spacer group to the monomers to form a side-chain. Consequently, the self-organising properties of liquid crystals can be used to influence the conducting polymer backbone and control the electrical, magnetic and mechanical properties of the material. Some applications of these interesting materials are discussed.
\end{abstract}

Keywords: liquid crystal, conducting polymer, mesogen, PPV, polythiophene, polypyrrole, polyaniline

\section{Introduction}

Polymers have generally been used in electrical and electronic applications as insulators, as they have localised electrons and are unable to provide electronic or ionic charge carriers to convey an electric current. Since the first report of a highly conducting polymer in 1977 by Shirakawa, Heeger and MacDiarmid ${ }^{1}$, these materials have attracted the interest of researchers and industrialists alike, as they show valuable electrical, electronic, magnetic and optical properties. The possibility of combining in single materials the light weight and processibility of organic polymers and the electronic properties of semiconductors, has been the driving force for numerous applications ${ }^{2-4}$.

Research into conducting polymers became exciting when a large increase in conductivity $\left(1 \times 10^{5} \mathrm{Sm}^{-1}\right)$ was observed in polyacetylene as a result of mild chemical treatment with iodine vapour ${ }^{5}$. Despite being the most highly-conductive polymers, polyacetylenes are easily degraded by oxidation and are sensitive to humidity. As a result, research was extended to polymers of aromatic and heterocyclic compounds such as thiophene, furan, pyrrole and aniline. The early applications of conducting polymers were restricted by their intractability and insolubility, although these drawbacks were partly resolved by the use of alkyl substitution, surfactant dispersion and other methods. The resulting polymers were effectively fusible and soluble, so they could be processed into useful films, fibres and moulded objects.

Liquid crystals have been commercially important for several decades, especially for applications in electronic displays and TV screens. Simple liquid crystal molecules often have a rod-like structure consisting of a rigid aromatic part (or mesogen) attached to a short flexible chain (spacer group). In between their normal (isotropic) liquid and crystalline states, they can exhibit well-known liquid crystalline mesophases such as those in Figure 1 ( $a$ and $b$ ), in which the molecules are very responsive to applied electric or magnetic fields. 
Figure 1. Typical molecular organisation in liquid crystals: (a) nematic; (b) smectic; (c) typical LC polymers with mesogenic groups (cylinders) and flexible spacers (zig-zags) built into their main chain (backbone), or into side-chains.

(a)

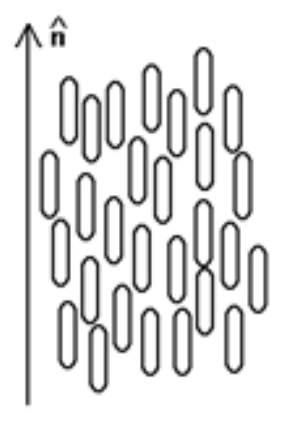

(b)

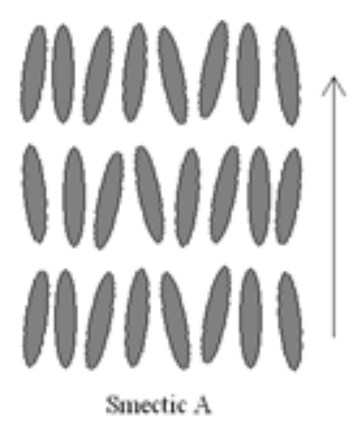

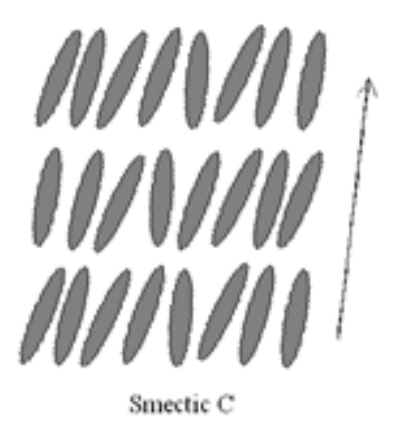
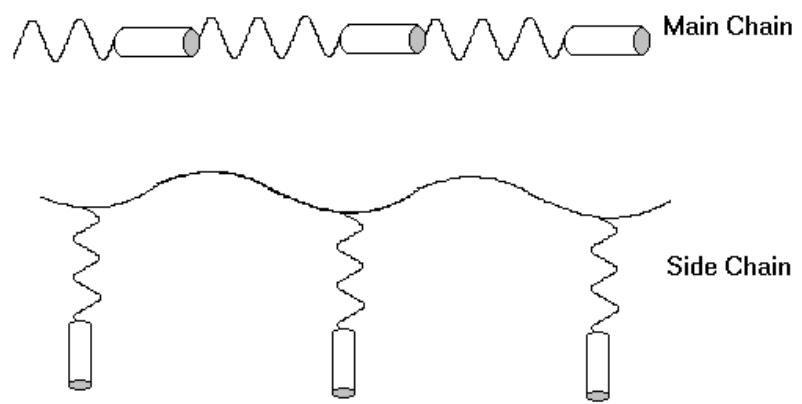

(c)

Polymer liquid crystals [simply represented in Fig. 1 (c)] have similar types of self-organisation, but owing to the relatively high viscosity of polymers, their response to external fields can be rather slow. Recently, side-chain liquid crystalline polymers (SCLCPs) have gained importance, due to having a wider phase transition temperature range and higher thermal stability than their small-molecule counterparts.

Among these materials, polysiloxane-based SCLCPs have received extensive attention because of the excellent physical properties of silicone polymers, such as high flexibility, weatherability, low surface tension and good water resistance. ${ }^{6}$ An example was reported by Wang et al. ${ }^{7}$, who synthesised a liquid crystalline polysiloxane with a grafted biphenyl mesogenic side-chain (Figure 2). Both the monomer and the grafted polymer had liquid crystalline properties, without any need for a large fraction of the siloxane units to have mesogenic substituents. 


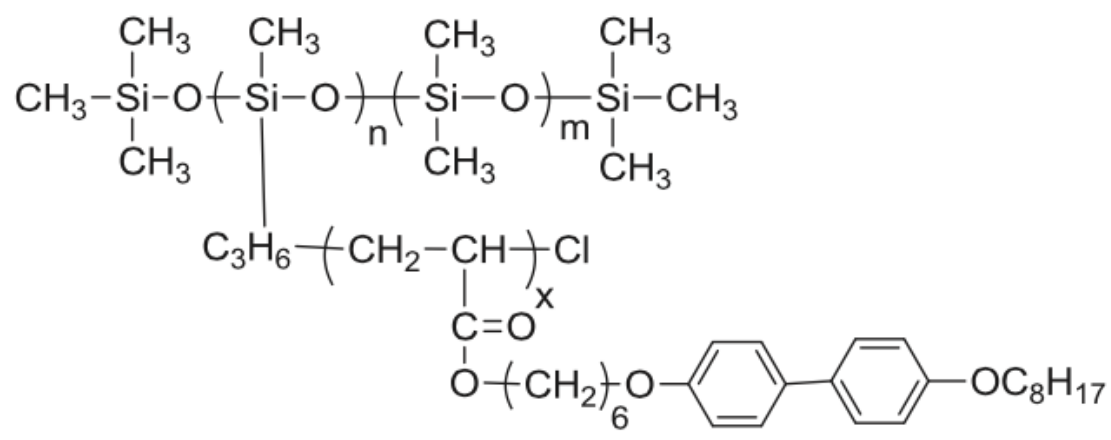

This article is mainly concerned with the properties of analogous conducting polymers, with a polarisable aromatic mesogen and a flexible aliphatic spacer group (typically 6 to 24 atoms long), grafted onto a conjugated $\left(\mathrm{sp}^{2}\right)$ carbon backbone. Because such a $\pi$-bonded backbone is much more rigid than a silicone one, this usually requires a LC moiety to be present on every monomer unit, in order that the LC groups can freely organise themselves and influence the polymer properties.

\section{Research on liquid crystalline conducting polymers}

Lambe et al. ${ }^{8}$, Akagi $^{9}$ and more recently Yang and $\mathrm{Hsu}^{10}$, have reviewed the synthesis and electro-optic applications of some main-chain and side-chain liquid crystal conductive polymers based on polypyrrole (PPY), polythiophene (PT), poly $(p$ phenylene vinylene) (PPV), polyfluorene (PF) and other conjugated backbones.

There has been a large amount of work on side-chain liquid crystalline conducting polymers since Akagi ${ }^{9}$ and co-workers first produced a LC polyacetylene in 1993. Much of the research on more stable aromatic polymers was pioneered in the UK, for example at Kingston University, where the first reported processible liquid crystal polyanilines $^{11}$ and polypyrroles ${ }^{12}$ were synthesised.

Gabaston et al. ${ }^{11}$ studied the synthesis and properties of liquid crystalline aniline monomers and polyanilines with mesogenic side-chains (Figure 3a). Their work showed that it was possible to control the conductivity of environmentallystable, semiconducting polymers by thermal treatment and the application of electric/magnetic fields. Both nematic and smectic mesophases were observed, and Figure $3 \mathrm{~b}$ is a polarised optical micrograph of the 3-ring ester polymer, showing its nematic structure at elevated temperature. 
Figure 3: (a) Precursors for the preparation of LC polyanilines. The liquid crystal side-chains were attached to nitrobenzenes, which were reduced to anilines and then polymerised to give SCLCCPs; (b) Typical nematic schlieren texture of one of the LC polymers observed under hot-stage polarised optical microscopy.

(a)<smiles>N#Cc1ccc(-c2ccc(OC(=O)c3ccc(OCCOc4ccccc4[N+](=O)[O-])cc3)cc2)cc1</smiles><smiles>[X]c1ccc(C)cc1</smiles>

(b)

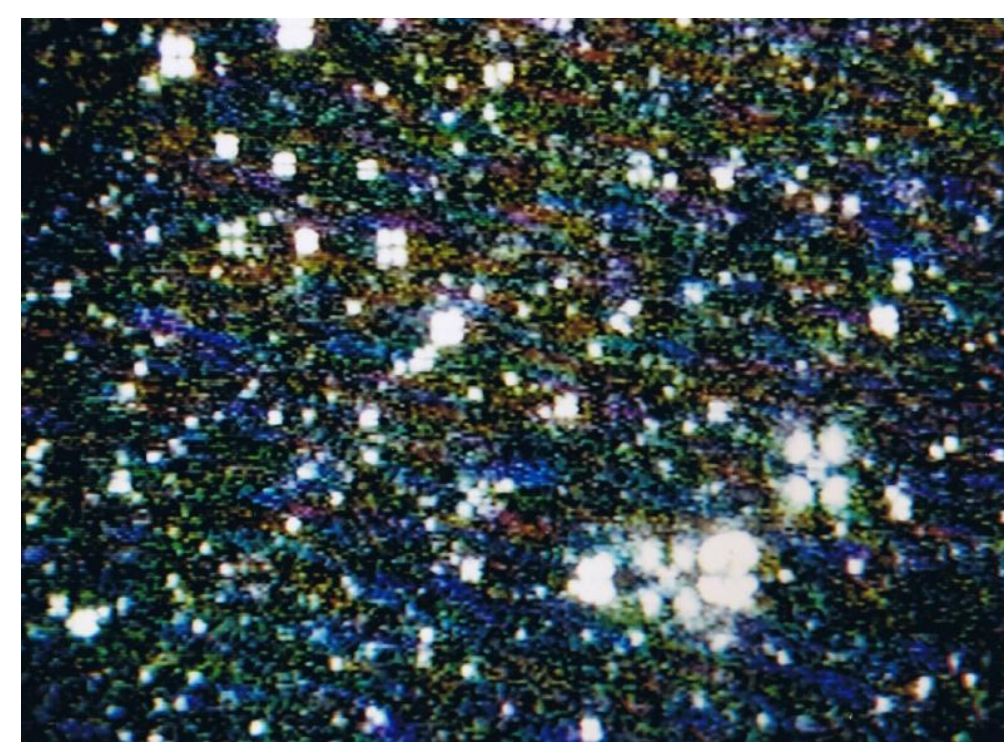

Ibison et al. ${ }^{12}$ reported the first soluble LC polypyrrole in 1995 , in which a hexyloxycyanobiphenyl side-chain was attached to the pyrrole heteroatom to make a symmetrical monomer unit. The polymer was a semiconductor whose bandgap and electrical properties changed on entering the nematic LC state. Later work ${ }^{13}$ demonstrated the production of nematic and smectic LC polypyrroles responsive to magnetic fields. 
Figure 4: (a) Polarised hot-stage microscope image for poly[N-nonyl-(cyanobiphenyloxy)pyrrole], suggesting a possible high-order smectic LC phase; (b) X-ray diffractograms of the same polymer; the initial amorphous polymer (magenta curve) transforms upon annealing (blue curve) into one showing peaks at low angle, indicating a large $(\sim 2.7 \mathrm{~nm})$ interlayer spacing in the smectic phase. [From Ref. 9(c).]

(a)

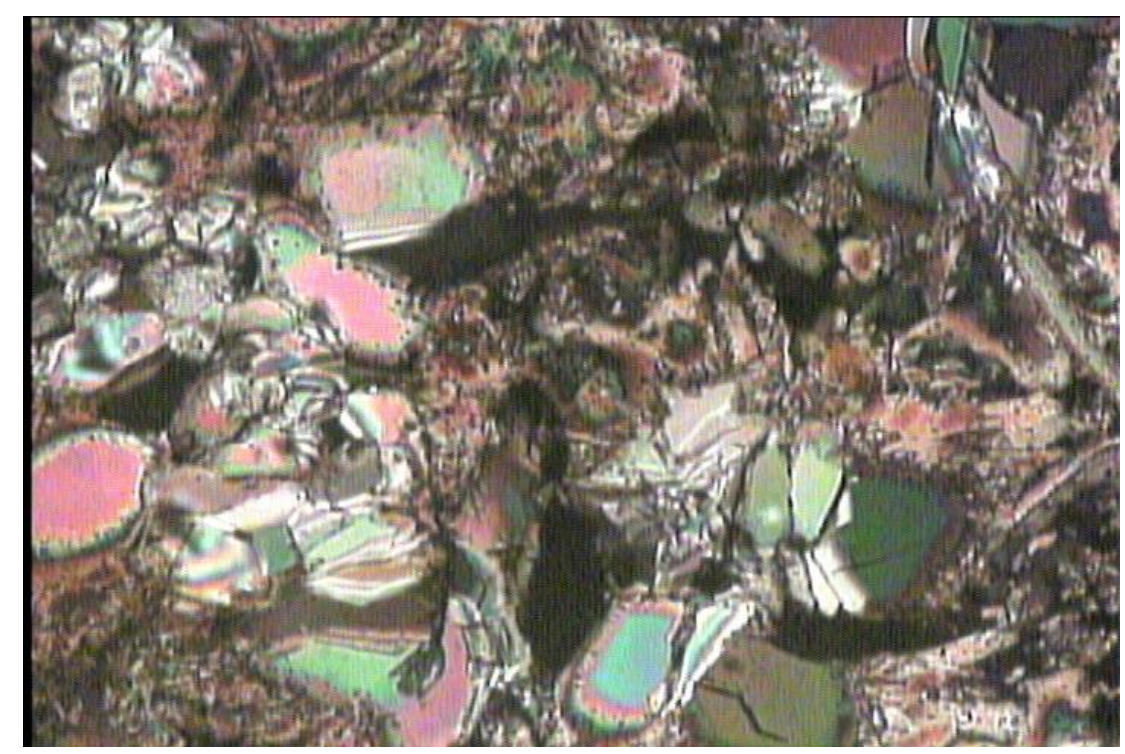

(b)

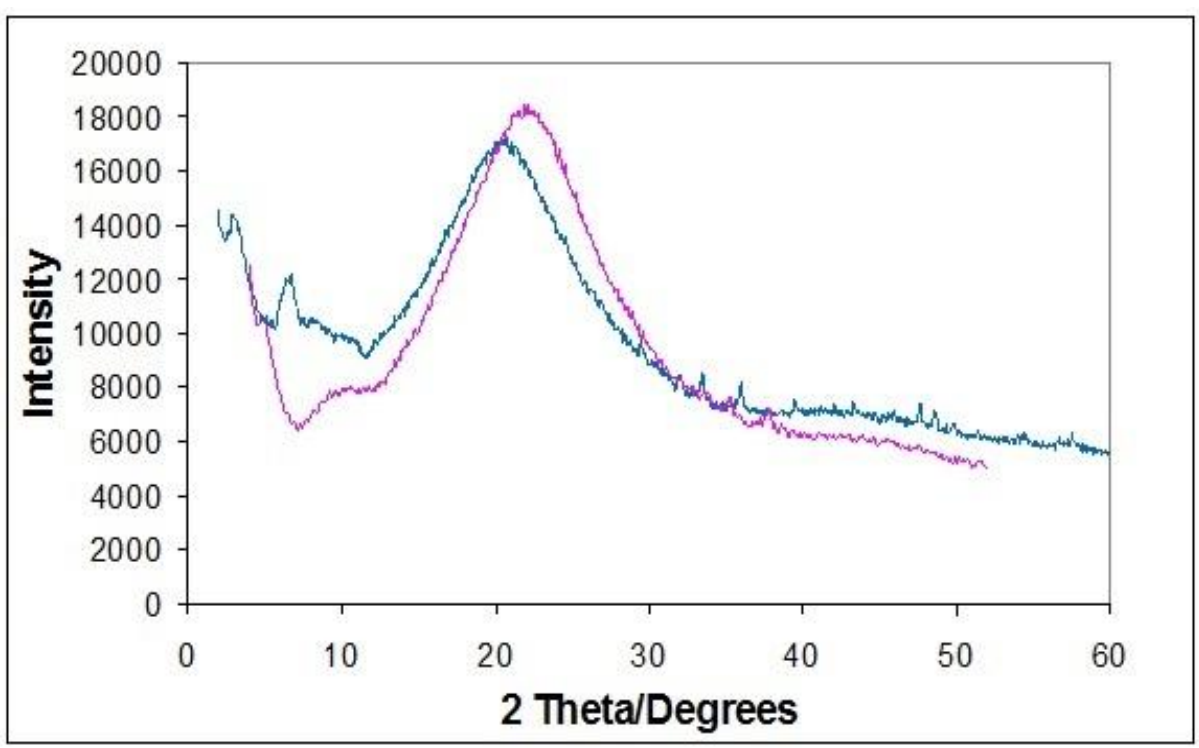

Hosseini et al. ${ }^{14}$ showed that in spite of the generally low processability of polypyrroles, they could synthesise a pyrrole polymer with an azo dye side group that conferred excellent solubility. The polymer was soluble in common organic solvents such as $\mathrm{CHCl}_{3}$, DMF and DMSO and was processible into a homogeneous thin film; in view of the biocompatibility of polypyrroles, these authors foresaw possible applications of the LC polymer in biological systems and related industries. A mosaic-type microscopic texture typical of smectic liquid crystal phases was observed under polarised light. 
Unsubstituted polythiophenes are conductive after doping, and have much better environmental stability than conducting polymers such as polyacetylene, but are intractable and infusible. This problem was first overcome by the incorporation of substituents at the 3-position of the thiophene ring, which not only furnished processable conducting polymers but also allowed the complete chemical and physical characterisation of the prepared materials ${ }^{15}$.

In similar work, Cho et al. ${ }^{16}$ and Chen et al. ${ }^{17}$ combined cyanobiphenyl mesogenic units with polythiophenes (Figure 5), in order to provide stable, selforganised liquid-crystalline conjugated materials. The orientation of cyanobiphenyl mesogen delivered polymer films with well-ordered morphologies and enhanced physical properties. After irradiation with UV light, the polymer films formed crosslinked networks, which not only enhanced the chemical stability, but also maintained the ordered nanostructure.

Figure 5: Incorporation of the mesogenic cyanobiphenyl onto polythiophenes.

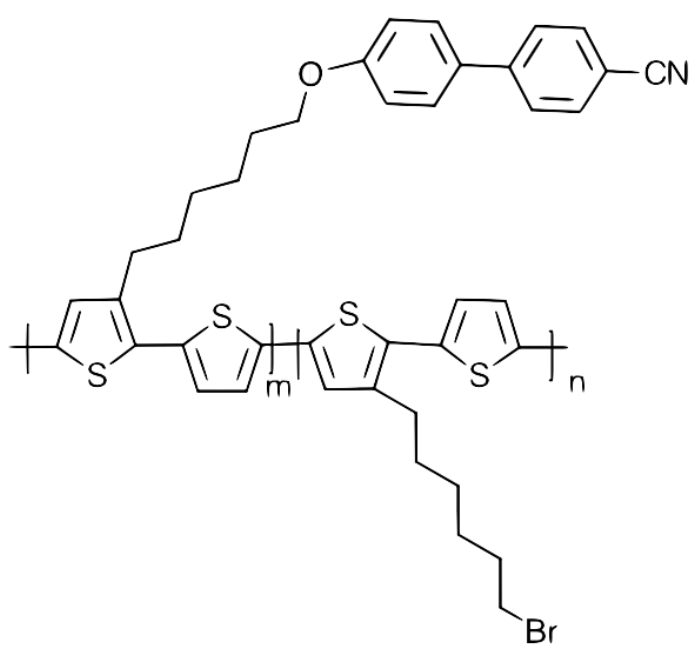

Zhao and co-workers ${ }^{18}$ synthesised a series of azobenzene-functionalised LC polythiophene derivatives (Figure 6). Their results indicated that these polymers had wide mesophase temperature ranges and good photoactivity. 


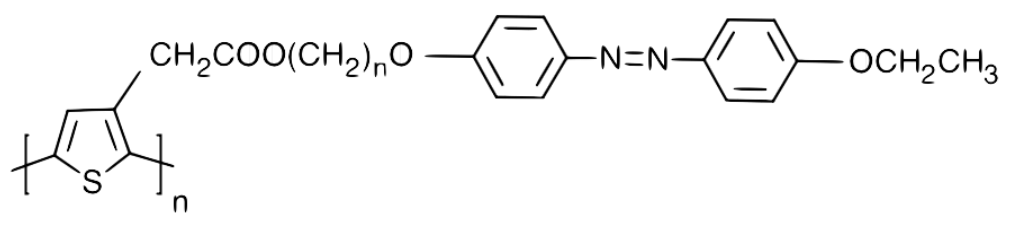

Armelin et al. ${ }^{19}$ synthesised a polythiophene derivative from a thiophene monomer with a malonic acid dimethyl ester substituent on its 3-position (Figure 7), using chemical oxidative-coupling polymerisation. They showed that the electrical conductivity of the new material, which was soluble in polar solvents except water, was higher than those typically found for poly(3-alkylthiophene) derivatives.

Figure 7: Malonic acid dimethyl ester-substituted polythiophene.<smiles>COC(=O)c1ccc(C(C)(C)C)s1</smiles>

Jin et al. ${ }^{20}$ prepared poly[3-(4-methoxy-4'-hexyloxybiphenyl)oxymethyl thiophene] (Figure 8) to examine the effect of liquid crystalline properties on the conducting polymer structure. The monomer showed an enantiotropic smectic liquid crystalline mesophase. A study of electrochemical polymerisation of the monomer by cyclic voltammetry showed a progressive increase in current with repeated cycling, demonstrating that the deposited polythiophene was highly conductive and increased the effective surface area of the electrode.

Figure 8: Poly[3-(4-methoxy-4'-hexyloxybiphenyl)oxymethylthiophene]<smiles>COCCOc1ccc(-c2ccc(OC)cc2)cc1</smiles>

We have seen that side-chains are an important component of some conducting polymers, and were originally introduced as the solubilising group. Both 
linear and branched alkyl side chains have been introduced into semiconducting polymers. While linear alkyl groups have been used for simple polymers such as poly(3-alkylthiophene)s, branched alkyl groups are often used in donor-acceptor polymer systems giving strong intermolecular interactions with solvents. Recent studies have shown that the use of branched alkyl groups is effective in altering the backbone orientation, while preserving good solubility and crystallinity. Osaka ${ }^{21}$ has recently summarised the influence of the main- and side-chain structures and the processing methods on the backbone orientation.

Subramaniyan et al. ${ }^{22}$ synthesised some novel and unusual thiazolothiazoledithienosilole copolymer semiconductors (Figure 9) bearing side-chains of various types, sizes and topologies. These polymers proved to be active in solar-cell devices, and the researchers studied the influence of the side-chains on their morphology, charge-transport and photovoltaic properties.

Figure 9: Thiazolothiazole-dithienosilole copolymers.

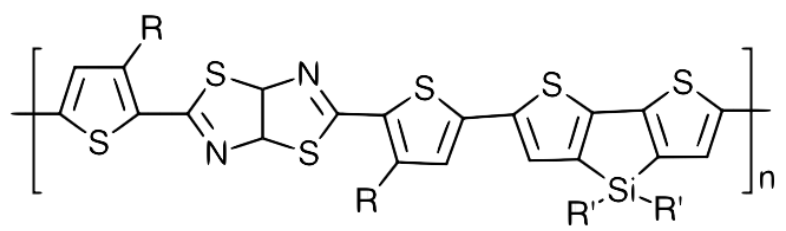

Somanathan ${ }^{23}$ studied the influence of fused polyaromatic side chains on the optical absorption and photoluminescence of polythiophene (Figure 10).

Figure 10: Structures of polythiophenes with polyaromatic side chains prepared in Ref. 23.

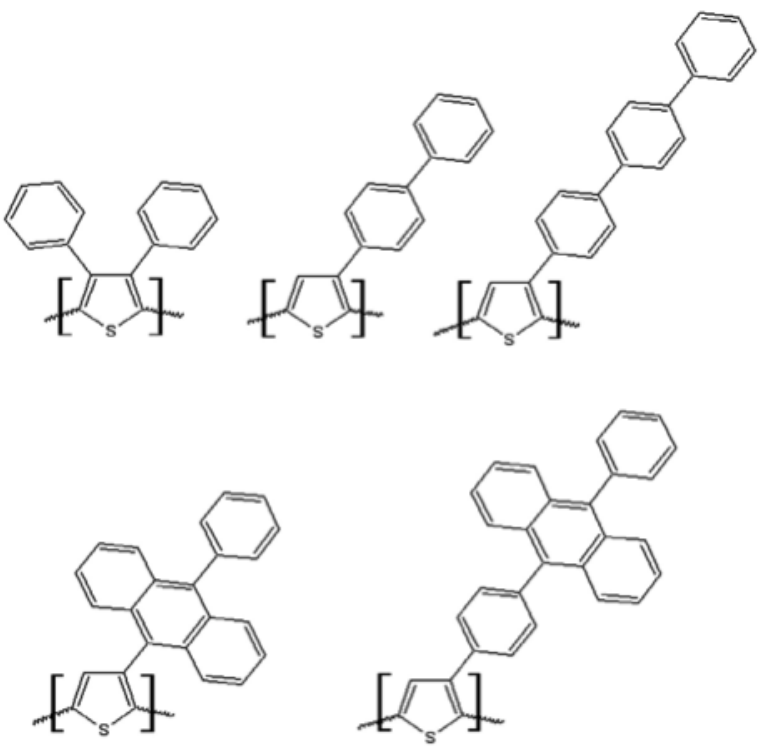


With an increase in the number of phenyl rings, the emission peak intensity increased and its wavelength was more red-shifted. The emission range extended from the UV to the visible range. By appropriately substituting the phenyl ring, the wavelength and intensity of the emission could be modified in polythiophenes. Selfpolarisation due to self-ordered molecular stacking helped to enhance the charge carrier mobility significantly ${ }^{23}$.

Soto et al. ${ }^{24}$ also synthesised 3-alkyloxymethylthiophenes substituted with a biphenyl group attached to alkyl chain spacers from 8 to 12 carbon atoms long (Figure 11). Differential scanning calorimetry (DSC) data for the monomers did not show any heat changes due to the presence of mesophases. The researchers also used polarised light microscopy, but no mesophase was observed: this was due to the insolubility and infusibility of their polymers, which was ascribed to the high molecular weight of the chains or to substantial crosslinking; hence the study was limited to electrical and thermal measurements.

Figure 11: 3-alkyloxybiphenylthiophenes.

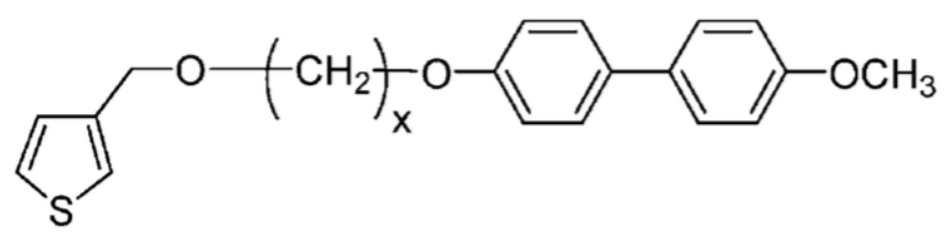

Yoon et al. ${ }^{25}$ synthesised a series of polythiophene derivatives with decyloligo(oxyethylene) side groups (PDnET) (Figure 12) by oxidative coupling polymerisation. As $\mathrm{n}$ was increased from 1 to 4 , the polymers with longer oligo(oxyethylene) units exhibited an increased tendency to form smectic liquid crystalline phases with well-ordered lamellar structures (except for $n=4$ ). Polymers with $n=1$ and $n=2$ had shorter oligo(oxyethylene) groups than PD3ET and formed less-ordered liquid-crystalline structures.

Figure 12: Poly(thiophene)s having decyl oligo(oxyethylene) side-chains.

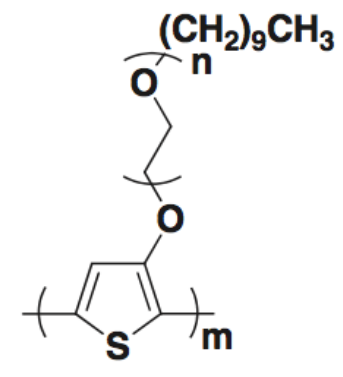

Brown et al. ${ }^{26}$ produced a novel thiophene-based conducting copolymer with mesogenic groups attached parallel to the conducting polymer backbone (Figure 13). They found that, after a non-mesogenic intermediate polymer had been converted into 
the hydrogen-bonded liquid crystalline polymer, there was a strong increase in the electronic conductivity (from $2.2 \times 10^{-5}$ to $1.4 \times 10^{-2} \mathrm{Sm}^{-1}$ ), due to the self-organising effect of the liquid crystal groups on the polythiophene backbone.

Figure 13: A thiophene-based LC copolymer with a laterally-attached mesogenic group ( $\mathrm{R}=\mathrm{n}$-hexyl).

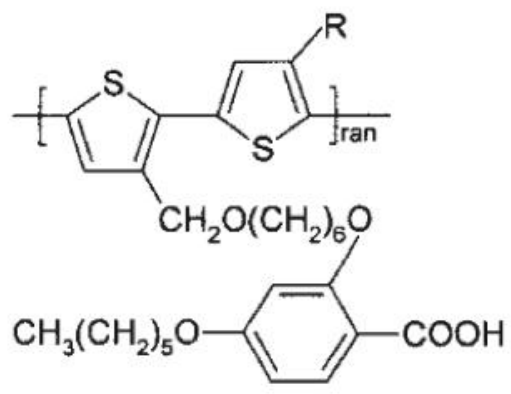

Many synthetic routes have been proposed to prepare poly( $p$-phenylene vinylene) derivatives ${ }^{10}$, which are of particular interest because of the strong luminescence of PPV. Attachment of long alkyl chains or mesogenic groups to the phenylene vinylene monomer unit improves the processibility of the resulting polymers in common organic solvents. Under polarised optical microscopy, most simple liquid crystalline PPV derivatives (such as those in Figure 14) show characteristic nematic textures with dark "brushes" when in their LC state.

Figure 14: Dialkoxy-PPV derivatives ( $R=$ a long alkoxy chain, such as $n-\mathrm{C}_{8} \mathrm{H}_{18} \mathrm{O}$ - or $n-\mathrm{C}_{6} \mathrm{H}_{14} \mathrm{O}-$ ).

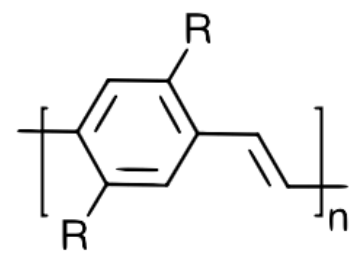

Several PPV derivatives such as poly(2,5-dioctyloxy-1,4-phenylenevinylene) (DOPPV) ${ }^{27}$ and poly(2,5,2',5'-tetrahexyloxy-7,8-dicyano-di-p-phenylenevinylene) (DHCNPPV), were found to exhibit nematic LC optical textures. X-ray observation of DO-PPV (Figure 15) after thermal annealing at $200^{\circ} \mathrm{C}$, revealed that the proportion of a lamellar LC phase substantially increased with temperature. DO-PPV and DHCNPPV both exhibited lamellar mesophases in the bulk ${ }^{10}$. 
Figure 15: XRD diffractograms of DO-PPV film annealed at $200^{\circ} \mathrm{C}$. Adapted with permission from Ref. 27 [Macromolecules, 2004, 37 (1), 181-186; DOI: 10.1021/ma035230n. Copyright (2004) American Chemical Society.]

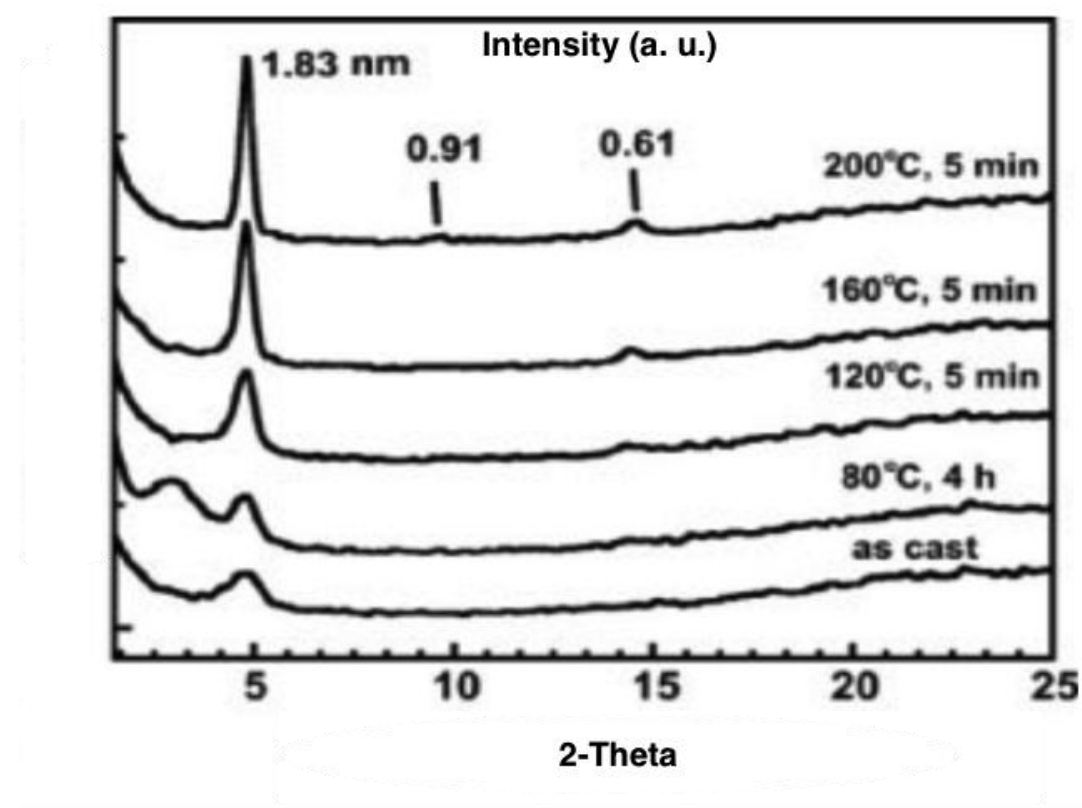

Onoda et al. ${ }^{28}$ synthesised poly(p-phenylene vinylene) derivatives substituted with a long side-chain containing alkyloxybiphenyl mesogenic units (Figure 16), and studied their electrical and optical properties. They confirmed the liquid-crystalline molecular alignment by optical microscopy and X-ray diffraction measurements. These polymers showed a typical optical texture for the liquid-crystalline phase and a sharp $\mathrm{X}$-ray diffraction peak due to the smectic layer stacking.

Figure 16: A poly(p-phenylene vinylene) with alkoxybiphenyl mesogenic unit ${ }^{28}$.<smiles>COc1cc(OCCCCCOc2ccc(-c3ccc(OCCCCCO)cc3)cc2)c(OC)cc1C=C(C)C</smiles>

The polycyclic aromatic rings of polyfluorene main chains can serve as the mesogenic units ${ }^{10}$, but this requires long or branched alkyl chains at the C-9 position of the fluorene to act as flexible spacers. While poly(9,9-dihexylfluorene) is amorphous, PFs with bulkier alkyl side-chains like poly(9,9-dioctylfluorene) or poly(9,9-bis(2ethylhexyl)fluorene) (Figure 17) display clear LC behaviour. 

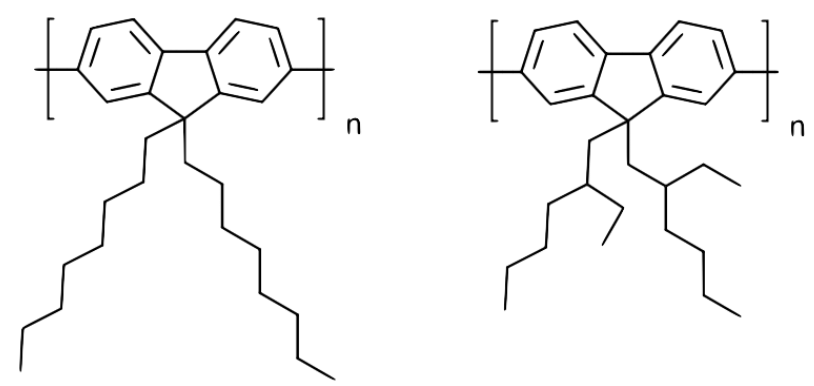

\section{Prospects for SCLCCP processing and applications}

In some senses, the backbones of conjugated polymers resemble poorly-insulated molecular "wires", with the potential for high conductivity along the molecular axis, and substantially lower in the perpendicular directions. Many such polymers can now be processed from solution or from the melt to make films, fibres or other products, but the molecular chains are often randomly arranged like spaghetti, so the inherent anisotropy of the polymers is not apparent. For applications in optoelectronics, conjugated polymers need to be chain-aligned, so that the mobility of the electronic charges can be maximised.

The molecular alignment of LC conjugated polymers can be achieved by direct rubbing of the active layer and by thermal annealing. These methods are usually performed in the LC temperature range, to allow self-organisation of the LC moieties. Rubbing the surface of such a material induces the alignment of mesogenic sidegroups along the rubbing direction, which favours the alignment of the polymer backbones approximately perpendicular to the rubbing direction.

A convenient and effective way to align LC conjugated polymers is the use of a magnetic field. Cho et al. ${ }^{29}$ synthesised poly(3,4-ethylenedioxythiophene) (PEDOT) films by electropolymerisation under an intense magnetic field onto ITO glass, in order to study the effects of the magnetic field on the electrochemical polymerisation of EDOT. By cyclic voltammetry, it was shown that the current was larger when the film was fabricated using magneto-electropolymerisation (1.17 Tesla). Increasing the electropolymerisation time also caused an increase in the current of the cyclic voltammogram, so the applied magnetic field sped up the polymerisation. SEM results also clearly revealed that the parallel applied magnetic field induced the formation of a sharper crystalline structure.

Lee, Kim et al. ${ }^{30}$ produced highly oriented conducting polyacetylene films by applying a strong magnetic field to a polymerisation system in a matrix of nematic liquid crystals. Oh, Akagi, Shirakawa et al. ${ }^{31}$ later demonstrated the alignment of an LC-substituted polyacetylene. Subsequently the molecules of many LC conjugated polymers have been magnetically aligned. For example, Figure 18 shows the polarised optical microscopic textures of a LC polythiophene ${ }^{32}$, before and after exposure to a relatively weak magnetic field $\left(0.2\right.$ Tesla) at $120^{\circ} \mathrm{C}$. There was a clear 
and reversible contrast, indicating a significant orientational effect, and the conductivity of the same polymer showed an anisotropy ratio of 2.4 under the same field.

Figure 18: Hot-stage polarised optical micrographs of a LC polythiophene (a) before and (b) after exposure to a magnetic field ${ }^{32}$.

(a)

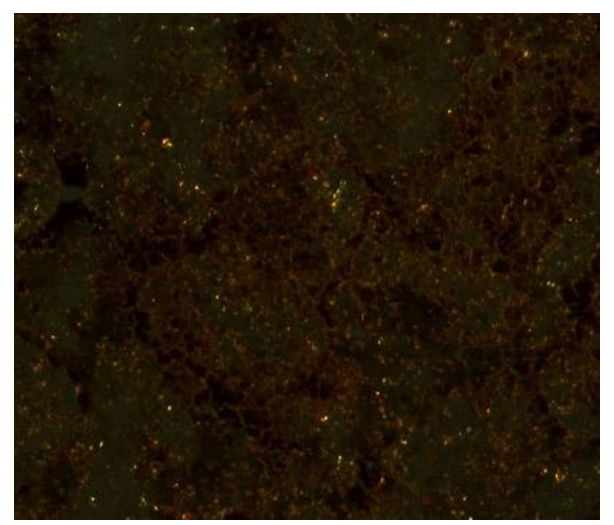

(b)

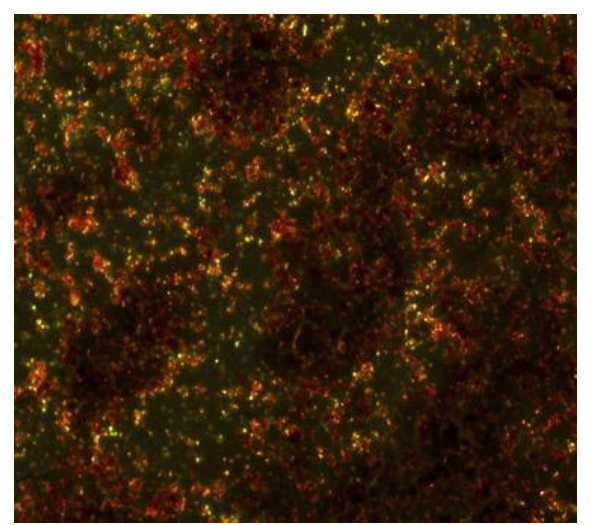

Brown et al. ${ }^{33}$ prepared liquid crystalline conducting polymers and demonstrated the first work on the synthesis of laser-alignable SCLCCPs. They established synthetic routes to three series of liquid crystal conducting polymers (based on pyrrole, thiophene and aniline monomers) (Figure 19) and reported the optimum conditions for polymer preparation.

Figure 19: Monomers of the pyrrole, thiophene and aniline LCCPs used to demonstrate laseralignment microprocessing ${ }^{33}$.<smiles>N#Cc1ccc(-c2ccc(OCCn3cccc3)cc2)cc1</smiles><smiles>COCc1ccsc1OCCOc1ccc(-c2ccc(C#N)cc2)cc1</smiles>

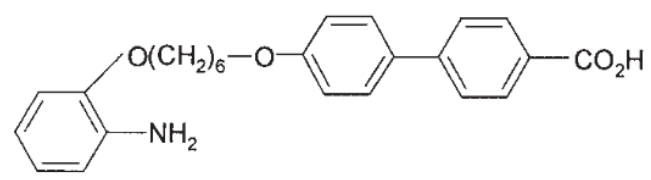

Solvent-cast films of these polymers had isotropic conductive properties, but after writing 50 fine, parallel lines on their surface with a focused laser beam, they showed increased conductivity in the direction of the laser scans (Table 1). 
Table 1: The apparent perpendicular $\left(\sigma_{\perp}\right)$ and parallel $\left(\sigma_{\|}\right)$conductivities for each of the laseraligned polymers, based on pyrrole (P1), thiophene (P2) and aniline (P3) monomers.

\begin{tabular}{|c|c|c|c|}
\hline Sample & $\sigma_{\perp} / \mathrm{Sm}^{-1}$ & $\sigma_{\|} / \mathrm{Sm}^{-1}$ & $\begin{array}{c}\text { Apparent } \\
\text { anisotropy }\end{array}$ \\
\hline $\mathrm{P} 1$ & $1.3 \times 10^{-6}$ & $4.0 \times 10^{-5}$ & 31 \\
\hline $\mathrm{P} 2$ & $5.7 \times 10^{-7}$ & $1.7 \times 10^{-6}$ & 3 \\
\hline $\mathrm{P} 3$ & $2.0 \times 10^{-5}$ & $4.0 \times 10^{-5}$ & 2 \\
\hline
\end{tabular}

The authors pointed out that the laser-aligned material represented less than $1 \%$ of the bulk. An estimate of the aligned volume for P1 suggested the local conductivity enhancement was 50,000 to 100,000 times. By using this thermo-optic alignment of SCLCCPs, it was subsequently confirmed that microscopic $(<3 \mu \mathrm{m})$ tracks of high conductivity could easily be written into comparatively insulating polymers by this means ${ }^{33}$.

Figure 20: Hot-stage polarised optical micrograph of a showing the effects of laser lithography on the LC polythiophene in Figure 19. (Line separation is $20 \mu \mathrm{m}$.

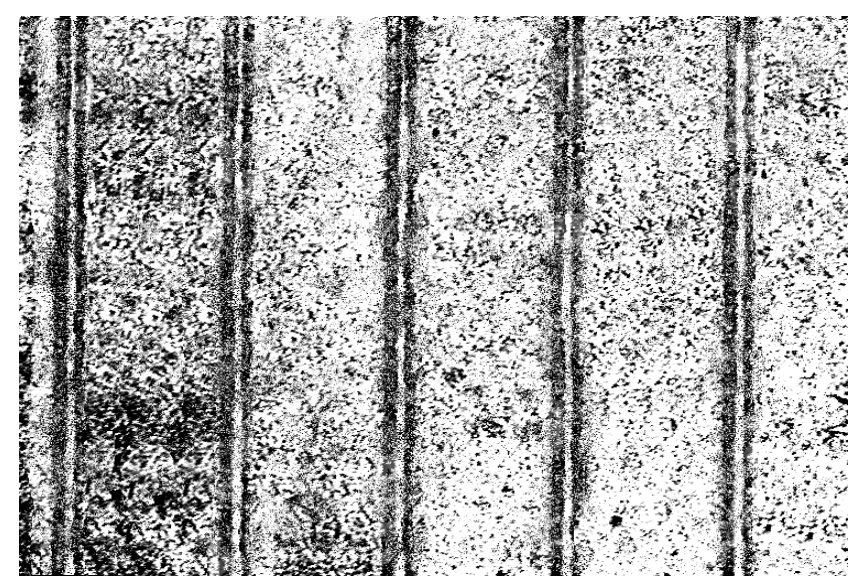

The field of liquid crystalline conducting polymers is of increasing importance, with the likelihood of a wide range of commercial uses. SCLCPs have potential applications in many fields, such as nonlinear optical devices and optical data storage ${ }^{34}$. LC derivatives of electroluminescent polymers such as poly(phenylene vinylene) have been chain-aligned and used to make polymer light-emitting devices (PLEDs) with polarised light emission. ${ }^{28}$

Several research groups have recently been exploring the potential of organised LCCP molecules to show high charge-carrier mobility. This parameter is important for the efficient operation of polymer-based transistors (OFETs) and photovoltaic solar cells. For example, McCullough et al. used LC poly(2,5-bis(3- 
alkylthiophen-2-yl)thieno[3,2-b]thiophene)s to make efficient and stable OFETs, in which the polymers showed carrier mobilities of up to $0.72 \mathrm{~cm}^{2} \mathrm{~V}^{-1} \mathrm{~s}^{-1}-$ much better than simple polythiophenes, and comparable to those of inorganic semiconductor films in conventional thin-film transistors. ${ }^{35}$

There is good deal of interest is plastic solar cells, with organic films as the active components, and several research groups have carried out extensive studies of LCCP-based devices ${ }^{8-10,16,17,22}$. Bulk heterojunction (BHJ) solar cells have been produced from polymers of the type shown in Figure 9. Very good performance [5.0\% PCE (average power conversion efficiency)] was shown by the polymers with branched alkyl side chains and perpendicular orientation of $\pi-\pi$ stacking to the substrate. Cells based on polymers with linear side chains and $\pi$-stacking parallel to the substrate had lower average efficiencies of 2.1-4.1\% PCE. Transient optical studies indicated that the differences in photovoltaic device performance correlated with the effectiveness of charge photogeneration and the influence of geminate electron-hole recombination ${ }^{22}$.

\section{Conclusion}

The ongoing research in the field of side-chain liquid crystalline conducting polymers has already shown that the organisation of the mesogenic moieties can affect the conductive backbones, and consequently improve their electrical, electronic and optical properties. Self-organising LCCPs are starting to achieve a commercial role in light-emitting devices, and in future, much more use is likely to be made of the use of electric, magnetic and thermo-optic effects for high-tech processing/microprocessing of these versatile organic semiconductors.

\section{References}

1. H. Shirakawa, E.J. Louis, A.G. MacDiarmid, C.K. Chiang and A.J. Heeger (1977). J. Chem. Soc., Chem. Commun., 578-580.

2. H.S. Nalwa (1997). Handbook of Organic Conductive Molecules and Polymers. John Wiley \& Sons, New York.

3. T.A. Skotheim, R.L. Elsenbaumer and J.R. Reynolds (1998). Handbook of Conducting Polymers. Marcel Dekker, New York, $2^{\text {nd }}$ Edition.

4. P.J.S. Foot and A.B. Kaiser (2004). "Conducting Polymers", in Kirk-Othmer Encyclopedia of Chemical Technology, John Wiley \& Sons, Inc., New York; DOI: 10.1002/0471238961.0512050318052514.a01.pub2.

5. B. Nordén (2000), Chairman of the Nobel Committee for Chemistry. Prize in Chemistry, 2000: Conductive Polymers.

6. T. Ganicz, T. Pakula, W. Fortuniak and E. Białecka-Florjanczyk (2005). Linear and hyperbranched liquid crystalline polysiloxanes. Polymer, 46, 11380-11388.

7. G. Wang, Y. Xiong and H. Tang (2015). Synthesis and characterization of a graft side-chain liquid crystalline polysiloxane. Journal of Organometallic Chemistry, 775, 50-54. 
8. G.J. Lambe, J.W. Brown and P.J.S. Foot (2006). Liquid Crystal Conducting Polymers, in "Specialty Polymers - Materials \& Applications”, ed. F. Mohammad, IK International Publishers, 2-18.

9. K. Akagi (2007). Synthesis and Properties of Liquid-Crystalline-Conjugated Polymers. Bull. Chem. Soc. Jpn., 80, 649-661.

10. S. Yang and C. Hsu (2009). Liquid Crystalline Conjugated Polymers and their Applications in Organic Electronics. Polymer Chemistry, 47, 2713-2733.

11. (a) L.I. Gabaston, P.J.S. Foot, J.W. Brown and V. Teissier (1994). New liquid crystalline conducting polymers and oligomers. Proc. Int. Conf. Liquid Crystal Polymers, Beijing, Sept. 1994; (b) L.I. Gabaston, P.J.S. Foot and J.W. Brown (1996). Synthesis and properties of liquid crystalline aniline monomers and semiconducting polyaniline with mesogenic side-chains. J. Chem. Soc., Chem. Commun., 429-430.

12. P. Ibison, P.J.S. Foot and J.W. Brown (1996). Proc. EMRS Spring Meeting 2015; Synth. Met. 76, 297-302.

13. (a) C T. Imrie and K. S. Ryder (2001). Pyrrole- and polypyrrole-based liquid crystals. J. Mater. Chem., 11, 990-995 ; (b) S. Abe, M. Kijima and H. Shirakawa (2001). Effect of mesogenic cores and length of spacers on liquid crystallinity of N-substituted polypyrrole derivatives. Synth. Met., 119, 421-422; (c) M. Almasri (2007). Liquid crystalline conducting polymers. Ph.D. Thesis, Kingston University.

14. S.H. Hosseini and M. Mohammadi (2009). Preparation and characterization of new polypyrrole having side-chain liquid crystalline moieties. Materials Science and Engineering, 29, 1503-1509.

15. H.S.O. Chan and S.C. Ng (1998). Synthesis, characterization and applications of thiophene-based functional polymers. Prog. in Polym. Sci., 1167-1231.

16. W. Cho, J. Wook Lee, Y. Gal, M. Kim and S. Jin (2014). Improved power conversion efficiency of dye-sensitized solar cells using side chain liquid crystal polymer embedded in polymer electrolytes. Materials Chemistry and Physics, 143, 904-907.

17. W. Chen, Y. Chen, F. Li, L. Chen, K. Yuan, K. Yao and P. Wang (2012). Ordered microstructure induced by orientation behavior of liquid-crystal polythiophene for performance improvement of hybrid solar cells. Solar Energy Materials \& Solar Cells, 96, 266-275.

18. X. Zhao, X. Hu, P.J. Zheng, L.H. Gan, C. Keat and P. Lee (2005). Synthesis and characterization of polythiophenes with liquid crystalline azobenzene as side chains. Thin Solid Films, 477, 88-94.

19. E. Armelin, O. Bertran, F. Estrany, R. Salvatella and C. Alemán (2009). Characterization and properties of a polythiophene with a malonic acid dimethyl ester side group. European Polymer Journal, 45, 2211-2221.

20. S. Jin, H. Lee, Y. Sun, H. Kim, K. Koh, Y. Gal and D. Park (1999). Synthesis and characterization of side chain liquid crystalline polymer with a polythiophene backbone. European Polymer Journal, 35, 89-94.

21. I. Osaka, K. Takimiya (2015). Backbone orientation in semiconducting polymers. Polymer, 59, A1-A15.

22. S. Subramaniyan, H. Xin, F. Kim, S. Shoaee, J.R. Durrant and S.A. Jenekhe (2012). Effects of Side Chains on Thiazolothiazole-Based Copolymer Semiconductors for High Performance Solar Cells. Adv. Energy Mater., 1, 854-860. 
23. N. Somanathan, B. Sambathkumar and S. Jeevarathinam Ananthakrishnan (2014). Studies on polythiophenes containing fused polyaromatic rings as side chains. European Polymer Journal, 52, 2331.

24. J.P. Soto, F.R. Diaz, M.A. del Valle, C.M. Nunez and J.C. Bernede (2006). Synthesis of new materials based on polythiophenes substituted with mesogen groups. European Polymer Journal, 42, 935-945.

25. Y. Yoon, T. Ko, J. Chung, J. Chung, J. Kim and J. Lee (2012). Liquid Crystalline Polythiophenes with Amphiphilic Side Chains. Macromol. Chem. Phys., 213, 285-292.

26. J.W. Brown, G.J. Lambe, P.J.S. Foot and J.A. Clipson (2004). Synthesis and Properties of a Novel Thiophene-Based Conducting Copolymer with Mesogenic Groups Attached Parallel to the Polymer Backbone. Macromol. Rapid Commun., 25, 1000-1003.

27. S.H. Chen, A.C. Su, S.R. Han et al. (2004). Molecular aggregation and luminescence properties of bul poly(2,5-di-n-octyl-1,4-phenylenevinylene). Macromolecules, 37 (1), 181-186.

28. M. Onoda, K. Tada, M. Ozaki and K. Yoshino (2000). An electroluminescent diode using liquidcrystalline conducting polymer. Thin Solid Films, 363, 9-12.

29. M.S. Cho, Y.Y. Yun, J.D. Nam, Y. Son and Y. Lee (2008). Effect of magnetic field on electrochemical polymerization of EDOT. Synth. Met., 158, 1043-1046.

30. Y.W. Lee, Y.S. Kim, Y.K. Lee, C.K. Park et al. (1987). Electronic structure of the polyacetylene film oriented by a liquid crystal solvent under magnetic field. Synth. Met. , 17(1-3), 539-544.

31. S.Y. Oh, K. Akagi, H. Shirakawa and K. Araya (1993). Synthesis and properties of liquidcrystalline polyacetylenes with a phenylcyclohexyl mesogenic moiety in the side-group. Macromolecules, 26 (23), 6203-6206.

32. D. Roudini (2015). Synthesis and Properties of Novel Thiophene-Based Liquid Crystalline Conducting Polymers. Ph.D. Thesis, Kingston University.

33. J.W. Brown, P.J.S. Foot, L.I. Gabaston, P. Ibison and A. Prevost (2004). Synthesis of LaserAlignable Liquid Crystalline Conducting Polymers. Macromol. Chem. Phys., 205, 1823-1828.

34. X. Li, L. Chen, Y. Chen, F. Li and Kai Yao (2012). Photocrosslinkable liquid-crystalline polythiophenes with oriented nanostructure and stabilization for photovoltaics. Organic Electronics, 13, 104-113.

35. I. McCulloch, M. Heeney, C. Bailey et al. (2006). Liquid-crystalline semiconducting polymers with high charge-carrier mobility. Nature Materials 5, 328-333. 